\title{
ON CELLS IN EUCLIDEAN SPACE THAT CANNOT BE SQUEEZED
}

\author{
ROBERT J. DAVERMAN ${ }^{1}$
}

1. Introduction. Let $K$ be a $k$-cell in Euclidean $n$-space $E^{n}$. Loosely speaking, we say that a map $f$ of $E^{n}$ onto itself squeezes $K$ to an $m$-cell provided that $f$ is a homeomorphism off $K$ and $f \mid K$ is related to a canonical projection of a round $k$-cell to a round $m$-cell. In case $n=3$ it is known that for each 3-cell $K$ in $E^{3}$ there exist many maps squeezing $K$ to 2-cells and many maps squeezing $K$ to 1-cells [6], and whenever $n \geqq 3$ it is known that for each 2-cell $D$ in $E^{n}$ there exist many maps squeezing $D$ to 1 -cells $([6],[7],[15])$. In this paper we point out counterexamples to generalizations of these results: there exists a $k$-cell $K$ in $E^{n}(3 \leqq k<n)$ for which there is no map squeezing $K$ to a lower dimensional cell, and there exists an $n$-cell $K^{*}$ in $E^{n}(n \geqq 4)$ for which there is no map squeezing $K^{*}$ to an $m$-cell $(m \leqq n-2)$. These counterexamples are embedded as everywhere wild subsets of $E^{n}$ with properties that easily eliminate the possibility of a squeezing map. However, this paper is not concerned primarily with such examples; instead, the purpose is to prove that for some relatively simple $k$-cells in $E^{n}(n \geqq 4)$, each one locally tame modulo a Cantor set, there is no map squeezing any one of them to either a 2-cell or a 1-cell.

2. Definitions. For each positive integer $k$ let $B^{k}$ denote the set $\left\{\left(x_{1}, \cdots, x_{k}\right) \in E^{k} \mid x_{1}{ }^{2}+\cdots+x_{k}{ }^{2} \leqq 1\right\}$. Clearly for $m \leqq k, B^{m}$ can be regarded as a subset of $B^{k}$. Let $\pi$ denote the projection map of $B^{k}$ to $B^{m}$ that sends $\left(x_{1}, \cdots, x_{k}\right)$ to $\left(x_{1}, \cdots, x_{m}\right)$.

Suppose $K$ is a $k$-cell in $E^{n}$. A map $f$ of $E^{n}$ onto itself is said to squeeze $K$ to an $m$-cell iff there exist homeomorphisms $g$ of $B^{k}$ onto $K$ and $h$ of $B^{m}$ onto $f(K)$ such that $f$ carries $E^{n}-K$ homeomorphically onto $E^{n}-f(K)$ and $f g=h \pi$. In particular, we say that such a map $f$ squeezes $K$ to the $m$-cell $f(K)$. Alternatively, if there is no map $f$ that squeezes $K$ to an $m$-cell, then we say that $K$ cannot be squeezed to an m-cell.

Received by the editors November 8, 1971 and, in revised form, February 23, 1972.

AMS (MOS) subject classifications (1970). Primary 57A15, 57A35, 57A45; Secondary 57A60.

${ }^{1}$ Research supported in part by NSF Grant GP 19966. 
A metric space $X$ is uniformly locally simply connected, or 1-ULC, iff to each $\epsilon>0$ there corresponds a $\delta>0$ such that any map from the boundary of the disk $B^{2}$ into a $\delta$-subset of $X$ can be extended to a map of $B^{2}$ into an $\epsilon$-subset of $X$. Similarly, given a subset $Y$ of $X$, we say that $Y$ is 1-ULC in $X$ iff to each $\epsilon>0$ there corresponds a $\delta>0$ such that each map of the boundary of $B^{2}$ into a $\delta$-subset of $Y$ can be extended to a map of $B^{2}$ into an $\epsilon$-subset of $X$.

Let $X$ be a compact subset of $E^{n}$. Following [1] we say that $X$ has Property $U V^{\infty}$ iff for each open set $U$ containing $X$ there exists an open set $V$ containing $X$ that is contractible in $U$. This concept has been studied by other authors (see [8] ) under a variety of names.

Given such a set $X$, we say that $X$ satisfies the Cellularity Criterion iff for each open set $U$ containing $X$ there exists a set $V$ containing $X$ such that each map of the boundary of $B^{2}$ into $V-X$ extends to a map of $B^{2}$ into $U-X$.

We use the symbols $B d$ and Int to denote the boundary and interior of a manifold-with-boundary, and we use $\mathrm{Cl}$ to denote topological closure.

For definitions of other terms used here the reader is referred to such papers as [3], [9], [10] .

3. Cells that cannot be squeezed to arcs.

Proposition 3.1. If $e$ is an embedding of $B^{k}$ in $E^{n}(n \geqq 3)$ such that $e\left(B^{k-1}\right)$ satisfies the Cellularity Criterion, then each $U V^{\infty}$ continuum $X$ in $e\left(B^{k-1}\right)$ also satisfies the Cellularity Criterion. Thus, $X$ is cellular provided $n \neq 4$.

Proof. Let $U$ be a neighborhood of $X$ in $E^{n}$. Since $X$ has property $U V^{\infty}$, there exists a closed neighborhood $N$ of $X$ in $e\left(B^{k-1}\right)$ that is contractible in $U \cap e\left(B^{k-1}\right)$. Define $Z=N \cap \mathrm{Cl}\left(e\left(B^{k-1}\right)-N\right)$. Use the structure of $e\left(B^{k}\right)$ to lift the induced contraction (obtained by restriction) of $Z$ off $X$, defining a contraction of $Z$ in $U \cap\left(e\left(B^{k}\right)-X\right)$. Apply Tietze's Extension Theorem to extend this contraction to one having domain a neighborhood $W_{2}$ of $Z$ in $E^{n}$ and range $U-X$.

Let $W_{1}$ be an open subset of $U$ such that $W_{1} \cap e\left(B^{k-1}\right)=N-Z$, and $W_{3}$ an open subset of $E^{n}$ containing $e\left(B^{k-1}\right)-N$ such that $W_{1} \cap W_{3}=\varnothing$. The hypothesis that $e\left(B^{k-1}\right)$ satisfies the Cellularity Criterion implies the existence of a neighborhood $V^{*}$ of $e\left(B^{k-1}\right)$ such that each loop in $V^{*}-e\left(B^{k-1}\right)$ is contractible in $\left(W_{1} \cup W_{2} \cup W_{3}\right)$ $-e\left(B^{k-1}\right)$. Define $V^{\prime}=V^{*} \cap W_{1}$.

We assume that if $k=n$, then $X \cap e\left(\operatorname{Bd} B^{k-1}\right) \neq \varnothing$, for otherwise the Corollary to Theorem 8 of [9], which applies to $U V^{\infty}$ continua as well as to compact absolute retracts, implies that $X$ is cellular. In 
this case there exists a neighborhood $V$ of $X$ in $E^{n}$ such that $V \subset V^{\prime}$ and each point of $V \cap\left(e\left(B^{k-1}\right)-X\right)$ can be joined to a point of $V \cap e\left(\mathrm{Bd} B^{k-1}\right)$ by an arc contained in $V^{\prime} \cap\left(e\left(B^{k-1}\right)-X\right)$; in case $k<n$ define $V=V^{\prime}$.

We show that any loop in $V-X$ is contractible in $U-X$. Let $f$ be a map of $\mathrm{Bd} B^{2}$ into $V-X$. Then $f$ is homotopic in $V^{\prime}-X$ to a map $f^{\prime}$ of $\mathrm{Bd} B^{2}$ into $V-e\left(B^{k-1}\right)$ : if $k=n$ we adjust $f$ slightly so that $f\left(\mathrm{Bd} B^{2}\right)$ meets $e\left(B^{k-1}\right)$ at just a finite number of points and perform a homotopy in $V^{\prime}-X$ that pushes each such point along an arc in $V^{\prime}-X$ out over the boundary of $e\left(B^{k-1}\right)$; if $k<n$ we can perform a slight adjustment of $f$ to move $f\left(\mathrm{Bd} B^{2}\right)$ away from $e\left(B^{k-1}\right)$. By hypothesis $f^{\prime}$ can be extended to a map

$$
F: B^{2} \rightarrow\left(W_{1} \cup W_{2} \cup W_{3}\right)-e\left(B^{k-1}\right) \subset\left(W_{1} \cup W_{2} \cup W_{3}\right)-X .
$$

However, $F\left(B^{2}\right)$ may contain points of $W_{3}$ outside $U$. To remedy this, remove the interiors of finitely many pairwise disjoint 2-cells in $B^{2}$ to obtain a disk with holes $H$ in $B^{2}$ such that

$$
\begin{gathered}
\mathrm{Bd} B^{2} \subset \mathrm{Bd} H, \quad F(H) \subset W_{1} \cup W_{2} \subset U, \\
F\left(\operatorname{Bd} H-\operatorname{Bd} B^{2}\right) \subset W_{2} .
\end{gathered}
$$

Redefine $F$ on each component $Y$ of $B^{2}-H$ by restricting the contraction of $W_{2}$ in $U-X$ to $\mathrm{Bd} Y$. This produces the required contraction of $f\left(\mathrm{Bd} B^{2}\right)$ in $U-X$. The second part of the proposition follows, of course, from [9, Theorem 1$]$.

Corollary 3.2. If $e$ is an embedding of $B^{k}$ in $E^{n}(n \geqq 5)$ such that $e\left(B^{k-1}\right)$ is cellular, then each Cantor set $C$ in $e\left(B^{k-1}\right)$ is tame.

Proof. Select an arc $X$ in $e\left(B^{k-1}\right)$ containing $C$. By the preceding proposition $X$ is cellular, and by [10, Lemma 3] $C$ is tame.

Corollary 3.2 also holds when $n=3$, in which case it is a direct consequence of McMillan's collapsing theorem [11, Theorem 1].

A compact 0 -dimensional subset $C$ of a cell $K$ is said to be tame relative to $K$ iff $C \cap \mathrm{Bd} K$ is tame in $\mathrm{Bd} K$ and $C \cap \operatorname{Int} K$ is locally tame in Int $K$. In addition, a 0 -dimensional $F_{\sigma}$-set $F$ in $K$ is said to be tame relative to $K$ iff $F$ can be expressed as a countable union of compact subsets that are tame relative to $K$.

Proposition 3.3. If $K$ denotes a $k$-cell in $E^{n}(3 \leqq k \leqq n, n \geqq 4)$ that is locally tame modulo a Cantor set $C$ and that can be squeezed to an arc, then there exists a 0-dimensional $F_{\sigma}$-subset $F$ of $K$ such that $F$ is tame relative to $K$ and $E^{n}-K$ is 1-ULC in $\left(E^{n}-K\right) \cup F$. 
Proof. Let $f$ be a map of $E^{n}$ onto itself that squeezes $K$ to an arc and $g: B^{k} \rightarrow K$ and $h: B^{1} \rightarrow f(K)$ the accompanying homeomorphisms, such that $f g=h \pi$. Enumerate the rational numbers in $(-1,1)$ as $r_{1}, r_{2}, \cdots$, and for $i=1,2, \cdots$ define a $(k-1)$-cell $Q_{i}$ as $g \pi^{-1}\left(r_{i}\right)=f^{-1} h\left(r_{i}\right)$. Since the nondegenerate point inverses under $f$ are $(k-1)$-cells like these $Q_{i}$ 's, it follows from [1, Lemma 5.2] that each $Q_{i}$ satisfies the Cellularity Criterion.

Case $1.3 \leqq k \leqq n-2$. By Corollary 3.2 each $Q_{i}$ is locally tame modulo the tame Cantor set $Q_{i} \cap C$. The dimension restriction for this case implies $\operatorname{dim} Q_{i} \leqq n-3$, from which one can show easily that $E^{n}-Q_{i}$ is 1-ULC. Thus, $Q_{i}$ is tame ([3, Theorem 2], [12, Theorem 1]). Then, for any map $s$ of $B^{2}$ into $E^{n}$ such that $s\left(\operatorname{Bd} B^{2}\right)$ $\subset E^{n}-K$, the map can be altered slightly, pushing $s\left(B^{2}\right)$ off the $Q_{i}$ 's one at a time, to define a map $s^{\prime}$ on $B^{2}$ such that (i) $s^{\prime} \mid \operatorname{Bd} B^{2}=$ $s \mid \mathrm{Bd} B^{2}$, (ii) $s^{\prime}$ is close to $s$, and (iii) $s\left(B^{2}\right) \cap K$ is a 0 -dimensional subset of $K-\bigcup Q_{i}$.

Essentially (up to a short homotopy) there are just countably many maps of $\mathrm{Bd} B^{2}$ into $E^{n}-K$ requiring extension. Thus, using the property established in the preceding paragraph, we can find a 0 -dimensional $F_{\sigma}$-set $F$ in $K-\cup Q_{i}$ such that $E^{n}-K$ is 1-ULC in $\left(E^{n}-K\right) \cup F$. Accordingly, the set $F$ can be decomposed into closed (relative to $K$ ) subsets $F_{1}, F_{2}, \cdots$, and since each $F_{j}$ misses $U Q_{i}$, Corollary 4 of [2] implies that $F_{j}$ is a subset of a Cantor set that is tame relative to $K$.

REMARK. Whenever $k<n-2$ we may assume that $F$ is a subset of $C$.

Case 2. $k=n$. Certainly $E^{n}-K$ is $1-$ ULC in $\left(E^{n}-K\right) \cup C$. Let $s$ denote a map of $B^{2}$ into $\left(E^{n}-K\right) \cup C$ such that $s\left(\operatorname{Bd} B^{2}\right) \subset$ $E^{n}-K$. Since $Q_{i} \cap C$ satisfies the cellularity criterion, $s$ can be modified near points of $s^{-1}\left(s\left(B^{2}\right) \cap Q_{i} \cap C\right)$ so that $s\left(B^{2}\right) \cap Q_{i} \cap C$ $=\varnothing$. But $Q_{i} \cap C$ is in a tame arc in $K$, which implies that $\mathrm{Bd} K-$ $\left(Q_{i} \cap C\right)$ is 1-ULC. Thus, the modification of $s$ can be chosen with range $\left(E^{n}-K\right) \cup\left(B d K-\left(Q_{i} \cap C\right)\right)$, and using the local tameness of $\mathrm{Bd} K-C$, we can improve this further to $\left(E^{n}-K\right) \cup\left(C-Q_{i}\right)$. Furthermore, by repeating this process carefully we find a map $s^{\prime}$ of $B^{2}$ such that (i) $s\left|\mathrm{Bd} B^{2}=s^{\prime}\right| \mathrm{Bd} B^{2}$, (ii) $s^{\prime}$ is close to $s$, and (iii) $s^{\prime}\left(B^{2}\right) \subset\left(E^{n}-K\right) \cup\left(C-\cup Q_{i}\right)$.

As in Case 1 , there exists an $F_{\sigma}$-set $F$ in $C-\bigcup Q_{i}$ such that $E^{n}-K$ is 1-ULC in $\left(E^{n}-K\right) \cup F$, and, by [2], $F$ can be expressed as the countable union of tame closed subsets.

Case 3. $k=n-1$. The argument for this case requires some tech- 
nical variations on the argument for Case 2 , and we leave it to the interested reader.

Proposition 3.4. Suppose $K$ is a $k$-cell in $E^{n}(n \geqq 3), F$ is a 0 dimensional $F_{\sigma}$-set in $K$ such that $F$ is tame relative to $K$ and $E^{n}-K$ is 1-ULC in $\left(E^{n}-K\right) \cup F, M$ is a $(k-1)$-cell spanning $K$, and $\epsilon>0$. Then there exists an $\epsilon$-push $\theta$ of $K$ onto itself such that each loop in $E^{n}-K$ is contractible in $E^{n}-\theta(M)$.

Proof. As in the proof of Proposition 3.3, each map $f$ of $\mathrm{Bd} B^{2}$ into $E^{n}-K$ can be extended to a map $g$ of $B^{2}$ into $E^{n}$ such that $g^{-1}\left(g\left(B^{2}\right) \cap K\right)$ is 0-dimensional. Then, since $E^{n}-K$ is 1-ULC in $\left(E^{n}-K\right) \cup F$, we perform modifications of $g$ near points of $g^{-1}\left(g\left(B^{2}\right) \cap K\right)$ to define a map $g^{\prime}$ of $B^{2}$ into $\left(E^{n}-K\right) \cup F$ that extends $f$.

To complete the argument we need only push $M$ off $F$ with an $\epsilon$-push of $K$. The set $F$ can be decomposed into compact sets $F_{1}, F_{2}, \cdots$ that are tame relative to $K$. We can construct a sequence $\left\{\boldsymbol{\theta}_{n}\right\}$ of pushes of $K$, where $\theta_{n}$ first pushes $\operatorname{Bd} M$ off $B d K \cap\left(\bigcup_{i=1}^{n} F_{i}\right)$ and then pushes Int $M$ off $\left(\bigcup_{i=1}^{n} F_{i}\right)$ and keeps the adjusted $B d M$ fixed, with sufficient care to guarantee that $\theta=\lim \theta_{n}$ is an $\epsilon$-push of $K$ and $\theta(M) \cap F=\varnothing$.

REMARK. In case $3 \leqq k \leqq n-2$, one can easily show that $E^{n}-\theta(M)$ is 1-ULC, which implies that $\theta(M)$ is tame ([3], [12]). In case $k=n \geqq 5$, if $M$ is locally tame at each point of $M \cap$ Int $K$, it is also possible to show that $E^{n}-\theta(M)$ is 1-ULC, and Theorem 9 of [13] implies that $\theta(M)$ is tame.

Propositions 3.3 and 3.4 combine to imply that the cells of [4] cannot be squeezed to arcs.

THEOREM 3.5. For $3 \leqq k \leqq n$ and $n \geqq 4$ there exists a $k$-cell in $E^{n}$ that is locally tame modulo a Cantor set but that cannot be squeezed to a 1-cell.

Proof. The $k$-cells $K$ described in [4] are locally tame modulo Cantor sets, but each contains a 2-cell $D$ (with $D$ in $\mathrm{Bd} K$ if $k=n$ ) such that there is no small push $\theta$ of $K$ onto itself such that every loop in $E^{n}-K$ is contractible in $E^{n}-\theta(D)$.

\section{The composition of squeezes.}

Proposition 4.1. If $f_{r}$ is a map of $E^{n}$ onto itself that squeezes the $r$-cell $R$ to the s-cell $S$ and $f_{s}$ is a map of $E^{n}$ onto itself that squeezes $S$ onto the t-cell $T$, then $f_{s} f_{r}$ squeezes $R$ onto $T$. 
Proof. The only problem occurs in showing that $f_{s} f_{r} \mid R$ is conjugate to the canonical projection of $B^{r}$ onto $B^{t}$. Let $\pi_{r}$ denote the projection of $B^{r}$ onto $B^{s}$ and $\pi_{s}$ the projection of $B^{s}$ onto $B^{t}$. First we establish the following claim:

Any homeomorphism $\lambda$ of $B^{s}$ onto itself extends to a homeomorphism $L$ of $B^{r}$ onto itself such that $\lambda \pi_{r}=\pi_{r} L$.

Each $b \in B^{r}$ can be uniquely represented as $b=(x, y)$ where $x \in B^{s}$ and $y$ is a $(r-s)$-tuple. Define

$$
L(b)=(\lambda(x), m(x) \cdot y)
$$

where $m(x)=\left[\left(1-|\lambda(x)|^{2}\right) /\left(1-|x|^{2}\right)\right]^{1 / 2}$. (It is to be understood that $m(x) \cdot y=0$ if $|x|=|\lambda(x)|=1$.) Verifying that $L$ is the required homeomorphism is routine and is left to the reader.

We now consider the proof of the proposition. Let $g_{r}: B^{r} \rightarrow R$ and $h_{s}: B^{s} \rightarrow S$ denote the homeomorphisms such that $f_{r} g_{r}=h_{s} \pi_{r}$, and let $g_{s}: B^{s} \rightarrow \mathrm{S}$ and $h_{t}: B^{t} \rightarrow T$ denote the homeomorphisms such that $f_{s} g_{s}=h_{t} \pi_{s}$. Using the claim above we find a homeomorphism $L$ of $B^{r}$ onto itself such that $\pi_{r} L=\left(h_{s}{ }^{-1} g_{s}\right) \pi_{r}$.

Define $g: B^{r} \rightarrow R$ as $g=g_{r} L$. Then

$$
\left(f_{s} f_{r}\right) g=f_{s} f_{r} g_{r} L=f_{s} h_{s} \pi_{r} L=f_{s} h_{s} h_{s}{ }^{-1} g_{s} \pi_{r}=f_{s} g_{s} \pi_{r}=h_{t} \pi_{s} \pi_{r} .
$$

Thus, $f_{r} f_{g}$ squeezes $R$ to $T$.

THEOREM 4.2. For $3 \leqq k \leqq n$ and $n \geqq 4$ there exists a $k$-cell in $E^{n}$ that is locally tame modulo a Cantor set and that cannot be squeezed to a 1-cell or a 2-cell.

Since any 2-cell in $E^{n}$ can be squeezed to a 1-cell ([5, Theorem 2], [7, Theorem 1], [15, Theorem 3]), Proposition 4.1 implies that no cell satisfying Theorem 3.5 can be squeezed to a 2 -cell.

\section{Cells that cannot be squeezed.}

Proposition 5.1. If $K$ is a $k$-cell in $E^{n}(3 \leqq k<n)$ and $f$ is a map of $E^{n}$ to itself squeezing $K$ to an $m$-cell $(m<k)$, then $K$ contains a 2-cell $D$ that satisfies the Cellularity Criterion. Thus, if $n \geqq 5$, then $K$ contains a cellular 2-cell.

Proof. In case $2 \leqq m<k$, then by [14, Theorem 3] or [15, Theorem 2] there exists a tame arc $A$ in Int $f(K)$. Certainly $A$ must satisfy the Cellularity Criterion, and consequently $f^{-1}(A)$ also must satisfy it [1, Lemma 5.2]. Let $g: B^{k} \rightarrow K$ and $h: B^{m} \rightarrow f(K)$ be homeomorphisms such that $f g=h \pi$. Note that $f^{-1}(A)=$ $g \pi^{-1} h^{-1}(A)$, which implies that $f^{-1}(A)$ is a $(k-m+1)$ cell. Since 
$(k-m+1) \geqq 2, f^{-1}(A)$ collapses to a 2 -cell $D$, and such a cell satisfies the Cellularity Criterion [11, Theorem 1]. As before, the second statement of the proposition follows immediately from [9, Theorem 1].

An analogous proof can be given for the following result about codimension 0 cells.

Proposition 5.2. If $K$ is an $n$-cell in $E^{n}(n \geqq 4)$ and $f$ a map of $E^{n}$ onto itself squeezing $K$ to an $m$-cell $(m \leqq n-2)$, then $\mathrm{Bd} K$ contains a 2-cell $D$ that satisfies the Cellularity Criterion. Thus, if $n \geqq 5$, Bd $K$ contains a cellular 2-cell.

These results immediately imply that the cells constructed in [5] satisfy the following theorem.

TheOREM 5.3. For $3 \leqq k<n$ there exists a $k$-cell in $E^{n}$ that cannot be squeezed to an $m$-cell $(m<k)$ and there exists an $n$-cell in $E^{n}$ that cannot be squeezed to a $j$-cell $(j \leqq n-2)$.

Proof. Examples are described in [5] of $k$-cells in $E^{n}$ such that for no 2-cell $D$ in $K$ (or in $\operatorname{Bd} K$ if $k=n \geqq 4$ ) is $E^{n}-D$ simply connected. In particular, no 2-cell $D$ in $K$ (or in $B d K$ ) satisfies the Cellularity Criterion.

Question. Can each $n$-cell in $E^{n}$ be squeezed to an $(n-1)$-cell?

\section{REFERENCES}

1. Steve Armentrout, Homotopy properties of decomposition spaces, Trans. Amer. Math. Soc. 143 (1969), 499-507. MR 42 \#8214.

2. J. L. Bryant, On embeddings with locally nice cross-sections, Trans. Amer. Math. Soc. 155 (1971), 327-332. MR 43 \#2721.

3. J. L. Bryant and C. L. Seebeck III, Locally nice embeddings of polyhedra, Quart. J. Math. Oxford Ser. (2) 19 (1968), 257-274. MR 38 \#2751.

4. R. J. Daverman, On the scarcity of tame disks in certain wild cells, Fund. Math. 79 (1973), 63-77.

5. - On the absence of tame disks in certain wild cells (to appear).

6. R. J. Daverman and W. T. Eaton, An equivalence for the embeddings of cells in a 3-manifold, Trans. Amer. Math. Soc. 145 (1969), 369-381. MR 40 \#3519.

7. —_ Each disk in $E^{n}$ can be squeezed to an arc (manuscript).

8. R. C. Lacher, Cell-like spaces, Proc. Amer. Math. Soc. 20 (1969), 598-602. MR 38 \#2754.

9. D. R. McMillan, Jr., A criterion for cellularity in a manifold, Ann. of Math. (2) 79 (1964), 327-337. MR 28 \# 4528.

10. - Taming Cantor sets in $E^{n}$, Bull. Amer. Math. Soc. 70 (1964), 706-708. MR 29 \# 1628.

11. _ Piercing a disk along a cellular set, Proc. Amer. Math. Soc. 19 (1968), 153-157. MR 36 \#3332. 
12. R. T. Miller, Approximating codimension three embeddings, Ann. of Math. (2) 95 (1972), 406-416.

13. C. L. Seebeck III, Collaring an $(n-1)$-manifold in an $n$-manifold, Trans. Amer. Math. Soc. 148 (1970), 63-68. MR 41 \#2692.

14. — Tame arcs on wild cells, Proc. Amer. Math. Soc. 29 (1971), 197-201. MR 43 \#6896.

15. R. B. Sher, Tame polyhedra in wild cells and spheres, Proc. Amer. Math. Soc. 30 (1971), 169-174. MR 43 \#6887.

UNIVERSity OF TENNesSEe, KNOXVILLE, TENNESSEE 37916 